\title{
Z-Portal Continuum Dark Matter
}

\author{
Csaba Csáki, ${ }^{1}$ Sungwoo Hong, ${ }^{1,2,3}$ Gowri Kurup $\odot,{ }^{1,4}$ Seung J. Lee $\odot,{ }^{5}$ Maxim Perelstein $\odot,{ }^{1}$ and Wei Xue ${ }^{6}$ \\ ${ }^{1}$ Department of Physics, LEPP, Cornell University, Ithaca, New York 14853, USA \\ ${ }^{2}$ Department of Physics, The University of Chicago, Chicago, Illinois 60637, USA \\ ${ }^{3}$ Argonne National Laboratory, Lemont, Illinois 60439, USA \\ ${ }^{4}$ Department of Physics, University of Oxford, Parks Road, Oxford OX1 3PJ, United Kingdom \\ ${ }^{5}$ Department of Physics, Korea University, Seoul 136-713, Korea \\ ${ }^{6}$ Department of Physics, University of Florida, Gainesville, Florida 32611, USA
}

(Received 26 July 2021; revised 20 December 2021; accepted 24 December 2021; published 23 February 2022)

\begin{abstract}
We examine the possibility that dark matter (DM) consists of a gapped continuum, rather than ordinary particles. A weakly interacting continuum (WIC) model, coupled to the standard model via a $Z$ portal, provides an explicit realization of this idea. The thermal DM relic density in this model is naturally consistent with observations, providing a continuum counterpart of the "WIMP miracle." Direct detection cross sections are strongly suppressed compared to ordinary Z-portal WIMP, thanks to a unique effect of the continuum kinematics. Continuum DM states decay throughout the history of the Universe, and observations of cosmic microwave background place constraints on potential late decays. Production of WICs at colliders can provide a striking cascade-decay signature. We show that a simple Z-portal WIC model provides a fully viable DM candidate consistent with all current experimental constraints.
\end{abstract}

DOI: 10.1103/PhysRevLett.128.081807

Introduction.-Strong evidence from cosmology and astrophysics points to the existence of dark matter (DM), which cannot be made up of standard model (SM) particles. Many viable DM models have been proposed. Among these, the weakly interacting massive particle (WIMP) DM [1] has been the leading paradigm for decades. WIMPs arise from well-motivated theoretical extensions of the SM, such as the neutralino DM in supersymmetry [2]. The WIMP paradigm naturally reproduces the observed DM density; moreover, the relic abundance of WIMPs is insensitive to the initial conditions of the Universe due to the thermal equilibrium between the DM and SM gases in the early Universe. However, experimental searches for nongravitational signatures of WIMPs have not found any positive evidence yet, leading to increasingly tight constraints on this idea [3]. For example, scalar Z-portal DM, one of the simplest WIMP models, is ruled out by several orders of magnitude [4] by direct detection experiments [5-7].

In this Letter, together with a companion paper [16], we propose a novel framework for DM, based on quantum field theories with a gapped continuum spectrum. Rather than ordinary particles, in such models DM consists of a mixture of states with a continuous mass distribution above a certain "gap" scale $\mu_{0}$. Within this framework, we focus

Published by the American Physical Society under the terms of the Creative Commons Attribution 4.0 International license. Further distribution of this work must maintain attribution to the author(s) and the published article's title, journal citation, and DOI. Funded by SCOAP ${ }^{3}$. on the "weakly interacting continuum" (WIC) scenario, where the gap $\mu_{0} \sim 100 \mathrm{GeV}$ is near the electroweak scale and the continuum DM interacts with the SM via weak interactions. We argue that such a WIC model will maintain all the attractive features of WIMPs, including the natural consistency with the observed DM abundance and insensitivity of relic density to initial conditions. At the same time the continuum nature of the DM leads to striking new phenomenological features [17]. In particular, the distinct kinematics of low-energy scattering of continuum states leads to strong suppression of direct detection rates, reviving the possibility of $Z$-portal DM. In this Letter, we focus on the $Z$-portal model as a simple and explicit realization of WIC paradigm. The second unique phenomenological feature is that throughout the history of the Universe, DM states continuously decay via $\operatorname{DM}\left(\mu_{1}\right) \rightarrow$ $\operatorname{DM}\left(\mu_{2}\right)+\mathrm{SM}$ processes. Late decays of this kind may leave observable effects in the cosmic microwave background (CMB) due to energy deposition during and after the recombination epoch. At collider, production of heavy DM states may lead to spectacular signatures as they cascade-decay to invisible states near the gap and SM particles with characteristic multiplicities and spectra.

Gapped continuum theories have been used in various contexts in particle and condensed matter physics, including applications of unparticles [31] to Higgs physics and the hierarchy problem [32-35], string theory [36], the fractional quantum Hall effect $[37,38]$ or the 2D Ising model [39]. The theoretical framework for quantitative studies of continuum DM is presented in detail in the 
companion paper [16]. There we described how to construct the Hilbert space of gapped continuum theories, gave formulas for calculating rates of scattering and decay involving continuum DM states, and discussed how to treat their equilibrium and nonequilibrium thermodynamics. We also presented an explicit model of Z-portal WIC, based on a warped 5D soft-wall geometry [40,41], and applied our formalism to calculate the DM relic density in this model. After a brief review of the Z-portal WIC model, this Letter focuses on its phenomenology. The main results of our analysis are summarized in Fig. 1: it is demonstrated that the Z-portal WIC model can reproduce the observed DM density while being fully consistent with current experimental constraints, including direct detection, $\mathrm{CMB}$, and collider data.

Z-portal WIC model.-The continuum DM is described by a "generalized free field" $\Phi$, with an effective Lagrangian

$$
S=\int \frac{d^{4} p}{(2 \pi)^{4}} \Phi^{\dagger}(p) \Sigma\left(p^{2}\right) \Phi(p) .
$$

Here, $\Phi$ is a complex, gauge-singlet Lorentz scalar. The spectral density $\rho$ can be obtained as

$$
\rho\left(\mu^{2}\right)=-2 \operatorname{Im} \frac{1}{\Sigma\left(\mu^{2}\right)} .
$$

In a gapped continuum theory, $\rho$ has continuous support above the gap scale $\mu_{0}^{2}$, and vanishes below that scale.

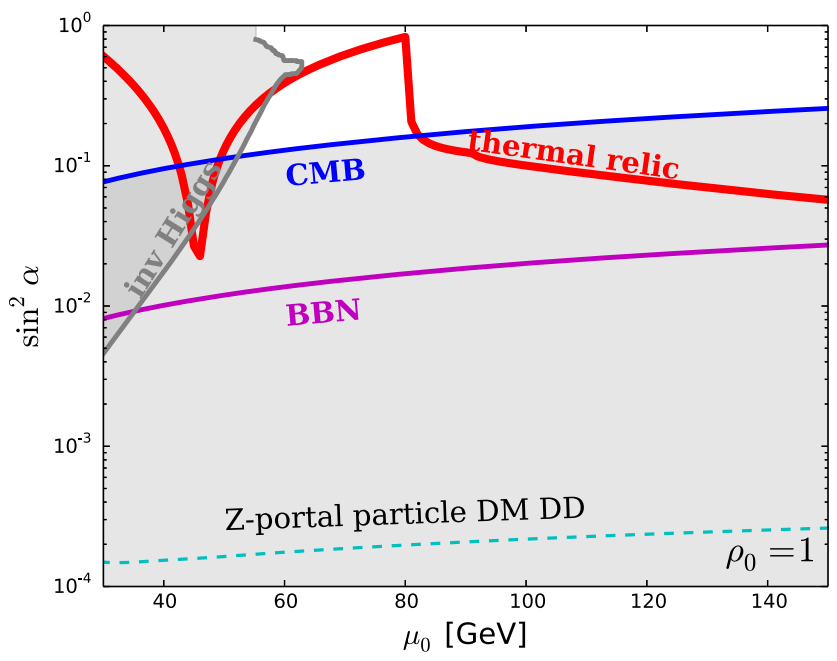

FIG. 1. Parameter space of the Z-portal WIC, with $\rho_{0}=1$. Red curve: thermal relic consistent with observations [8]. Space below the blue curve is ruled out by the CMB constraint $[9,50]$, while space below the magenta curve is also ruled out by the $\mathrm{BBN}$ constraint on electromagnetic energy injection [10-15]. Space above the gray curve is ruled out by the LHC constraint on exotic Higgs decays (with $m_{\chi}=500 \mathrm{GeV}$ ). For comparison, the bound of DM direct detection for Z-portal particle DM is shown as the dotted cyan curve.
Physically, the continuous parameter $\mu$ plays the role of the DM state mass. The singly excited sector of the Hilbert space consists of states $\left|\mathbf{p}, \mu^{2}\right\rangle$, and the function $\rho\left(\mu^{2}\right)$ is the density of states with respect to $\mu^{2}$. If the continuum arises from a known 5D theory, the spectral density can be calculated. The behavior of $\rho$ in the vicinity of the gap scale is especially important for DM phenomenology, since during thermal freeze-out and throughout the subsequent evolution of the Universe up to our own epoch, most of the DM states are clustered near the gap (see Supplemental Material, Sec. 1 [49]). It was shown in [16] that in a broad range of models, the spectral density near the gap takes the generic form

$$
\rho\left(\mu^{2}\right)=\frac{\rho_{0}}{\mu_{0}^{2}}\left(\frac{\mu^{2}}{\mu_{0}^{2}}-1\right)^{1 / 2},
$$

where $\rho_{0}$ is an order-one dimensionless constant. As discussed in [16], the value of $\rho_{0}$ is model dependent and is fixed by the normalization of a brane-to-brane propagator in the corresponding 5D theory. In Fig. 1 we show constraints for $\rho_{0}=1$ as a benchmark. To illustrate the impact of varying this parameter, the constraint plot for $\rho_{0}=2 \pi$ is included in the Supplemental Material [49].

To couple $\Phi$ to the SM sector, a new complex scalar $\chi$ is introduced, which is an $\mathrm{SU}(2)$ doublet and carries a $\mathrm{U}(1)_{Y}$ charge, $Y=-\frac{1}{2}$. Both $\Phi$ and $\chi$ are odd under a $Z_{2}$ symmetry responsible for DM stability, while all SM fields are even. The field $\chi$ has a canonical kinetic term and $m_{\chi}^{2}>0$. An interaction term

$$
\mathcal{L}_{\text {int }}=-\lambda \Phi \chi H+\text { H.c. }
$$

leads to mass mixing of $\chi$ with the continuum after electroweak symmetry breaking. Assuming $m_{\chi} \gg \mu_{0}$ and integrating out $\chi$ yields an effective Lagrangian coupling the continuum to SM:

$$
\begin{aligned}
\mathcal{L}_{\Phi-Z, W}= & \sin ^{2} \alpha\left[-\frac{i \sqrt{g^{2}+g^{\prime 2}}}{2}\left(\partial_{\mu} \Phi^{\dagger} \Phi-\Phi^{\dagger} \partial_{\mu} \Phi\right) Z^{\mu}\right. \\
& \left.+\frac{g^{2}+g^{\prime 2}}{4} \Phi^{\dagger} \Phi Z_{\mu} Z^{\mu}+\frac{1}{2} g^{2} \Phi^{\dagger} \Phi W_{\mu}^{+} W^{-\mu}\right] .
\end{aligned}
$$

Here, $g$ and $g^{\prime}$ are the $\mathrm{SM} \mathrm{SU}(2)_{L}$ and $\mathrm{U}(1)_{Y}$ gauge couplings, and the mixing angle $\alpha$ is given by

$$
\tan 2 \alpha=\frac{\sqrt{2} \lambda v}{m_{\chi}^{2}-\mu^{2}} .
$$

These interactions are responsible for WIC annihilation and decay. Note that the total number of the DM continuum states is conserved, even though they can decay into each other. The physics of WIC is described in terms of just three new parameters (the gap scale $\mu_{0}$, the mixing angle $\alpha$, and 
the spectral density normalization $\rho_{0}$ ), making for a simple and predictive model.

Boltzmann equations and thermal relic.-A dilute gas of continuum DM states is described by the occupation number $f(\mathbf{p}, \mu)$. In thermal equilibrium, the occupation number follows the usual Fermi-Dirac or Bose-Einstein distribution, with particle mass $m$ replaced by the continuum state mass $\mu$. The number density of continuum states is given by

$$
n=\int \frac{d \mu^{2}}{2 \pi} \nu\left(\mu^{2}\right)
$$

where

$$
\nu\left(\mu^{2}\right)=\rho\left(\mu^{2}\right) \int \frac{d^{3} p}{(2 \pi)^{3}} f(\mathbf{p}, \mu)
$$

is the DM mass distribution function. When considering the freeze-out of continuum annihilation to the SM, the Boltzmann equation for the continuum has the usual form (see detailed derivation in [16]),

$$
\frac{\partial n}{\partial t}+3 H n=-\langle\sigma v\rangle\left(n^{2}-n_{\mathrm{eq}}^{2}\right)
$$

where $n_{\mathrm{eq}}$ is the number density in thermal equilibrium. The thermal relic density for WIC is the same as for WIMP with the same thermally averaged cross section $\langle\sigma v\rangle$, but for WIC $\langle\sigma v\rangle$ includes averaging over $\mu^{2}$. Taking into account the Boltzmann factor and the form of the spectral density near the gap, Eq. (3), it is easy to see that the continuum distribution at temperatures below $\mu_{0}$ is localized near the gap scale. The WIC relic density is then the same as for a WIMP of mass $m=\mu_{0}$ and interactions in Eq. (5), up to small corrections of order $\mathcal{O}\left(T^{2} / \mu_{0}^{2}\right)$. Note that the thermal relic density does not depend on $\rho_{0}$, since the normalization of the spectral density cancels out in the $\langle\sigma v\rangle$.

The dominant WIC annihilation process during freezeout depends on the gap scale $\mu_{0}$. In the regime of $\mu_{0}<m_{W}$, the dominant channel is $\Phi \Phi^{*} \rightarrow Z \rightarrow f \bar{f}$. This process is $p$ wave, since the parity of the initial state is -1 . For a larger gap scale, $\mu_{0} \gtrsim m_{W, Z}, \quad s$-wave annihilations into pairs of vector bosons become relevant, $\Phi \Phi^{*} \rightarrow W^{+} W^{-}$and $\Phi \Phi^{*} \rightarrow Z Z$. In Fig. 1, the red curve corresponds to the observed DM relic abundance. The required value of the mixing angle $\sin ^{2} \alpha$ drops near the $Z$ pole, $\mu_{0} \simeq m_{Z} / 2$, where the cross section is enhanced by the $Z$-pole resonance. The decrease of the required $\sin ^{2} \alpha$ above $m_{W}$ is due to opening of new annihilation channel. Note that we do not include the WIC annihilations to one on-shell and one offshell $W$, which may give order-one corrections to the total annihilation rate in a narrow region of $\mu_{0}$ just below $m_{W}$.
Late decay and CMB constraints.-One of the main distinguishing features of WIC DM compared to ordinary particle DM is that throughout the history of the Universe, the continuum states are continuously decaying to the SM particles, $\Phi\left(\mu_{1}\right) \rightarrow \Phi\left(\mu_{2}\right)+$ SM. These processes are always unavoidable even in the presence of an exact $Z_{2}$ symmetry, due to the off-diagonal couplings among the continuum states such as in the interactions in Eq. (5). These decays keep dumping energy into the SM sector with $E \simeq \mu_{1}-\mu_{2}$, and can leave imprints during and after the recombination epoch.

The evolution history of the continuum states can be summarized as follows. Before the freeze-out they are in thermal and chemical equilibrium. After the WIC freezeout, which occurs at $T \sim \mu_{0} / 10$, the quasielastic scattering $\Phi+\mathrm{SM} \leftrightarrow \Phi+$ SM and (inverse) decays $\Phi \leftrightarrow \Phi+$ SM maintain kinetic equilibrium of the continuum with the SM particles and chemical equilibrium among the continuum states themselves. This forces the mass distribution of the continuum states to peak closer to the gap scale $\mu_{0}$ as the SM plasma temperature decreases. At temperature $T$, a typical DM state has mass $\mu$ such that $\Delta \mu \equiv \mu-\mu_{0} \sim T$. Eventually, the rate of these processes falls below the Hubble rate, the WICs fully decouple from the SM, and the chemical equilibrium among the continuum states can no longer be maintained. For typical parameters in the $Z$-portal WIC model, this decoupling occurs at $T_{d} \sim 0.1-1 \mathrm{GeV}$. After decoupling, the WIC mass distribution continues to evolve due to out-of-equilibrium WIC decays $\Phi \rightarrow \Phi+$ SM, with typical masses tending ever closer to the gap scale. This evolution is illustrated in Fig. 2, which is based on a numerical solution of the Boltzmann equation (see Supplemental Material [49]). An upper bound on the DM mass $\mu$ at time $t$ in this epoch can be estimated by equating the total decay rate of $\Phi(\mu)$ with the Hubble

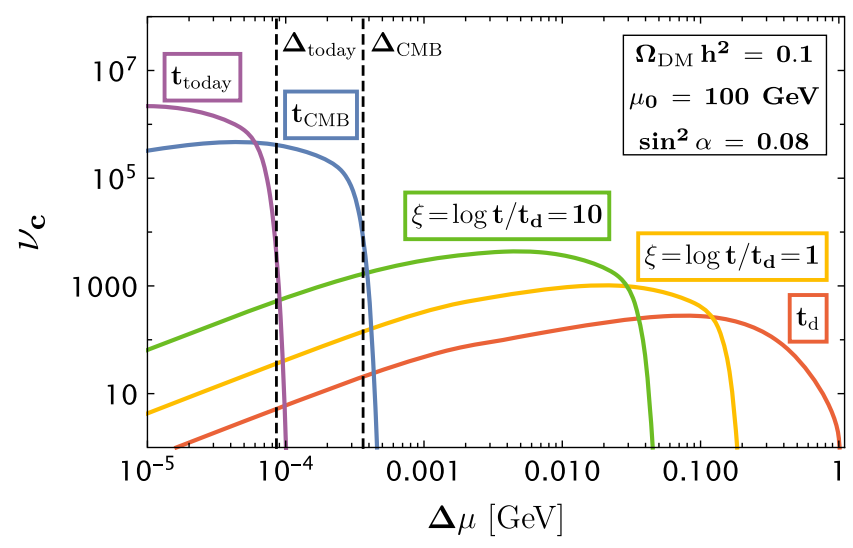

FIG. 2. Time evolution of the DM comoving number density $\nu_{c}$, as a function of mass above the gap $\Delta \mu=\mu-\mu_{0}$, after its kinetic decoupling from the SM at time $t_{d}$. The black lines indicate $\Delta_{\mathrm{CMB}}$ and $\Delta_{\text {today }}$, found analytically by setting $\Gamma=H$ at photon decoupling and today, respectively. The $y$-axis units are arbitrary. See Supplemental Material, Sec. 1 [49] for details. 
rate $H(t)$, since heavier DM states would have already decayed. This estimate agrees well with the numerical results, see Fig. 2. A typical DM mass at any given time is within a factor of a few from this bound, which implies that each DM state on average undergoes $\mathcal{O}(1)$ decays per Hubble time.

In the $Z$-portal WIC model, the DM states decay through an off-shell $Z, \Phi\left(\mu_{1}\right) \rightarrow \Phi\left(\mu_{2}\right)+Z^{*} \rightarrow \Phi\left(\mu_{2}\right)+f \bar{f}$. The rate of this decay (integrated over $\mu_{2}$ ) is given by

$$
\begin{aligned}
\Gamma(\Phi \rightarrow \Phi+f \bar{f})= & \frac{16 \sqrt{2} \rho_{0}}{15 \times 9009 \pi^{4}} \sin ^{4} \alpha \frac{\left(g^{2}+g^{\prime 2}\right)^{2}}{m_{Z}^{4}} \\
& \times\left(g_{A}^{2}+g_{V}^{2}\right)(\Delta \mu)^{5}\left(\frac{\Delta \mu}{\mu_{0}}\right)^{3 / 2} \\
\equiv & \Gamma_{0}\left(\frac{\Delta \mu}{\mu_{0}}\right)^{13 / 2},
\end{aligned}
$$

where $\Delta \mu=\mu_{1}-\mu_{0}$ and $g_{A, V}$ are the SM $Z$ couplings. The strong dependence on the DM mass arises from three-body phase space $\propto(\Delta \mu)^{3}$, the matrix element-squared $\propto(\Delta \mu)^{2}$, and final state spectral density integration $\propto\left(\Delta \mu / \mu_{0}\right)^{3 / 2}$. Here, we assumed $\Delta \mu \gg m_{f}$; the rate rapidly drops to zero near the kinematic threshold $\Delta \mu=2 m_{f}$.

For typical WIC parameters, $\Delta \mu$ drops below muon and pion thresholds soon after kinetic decoupling, while the decays to $e^{+} e^{-}$and the three neutrino flavors continue at late times. The first of these decays is potentially problematic for phenomenology, since it injects EM energy which can reionize hydrogen atoms after recombination. For example, if $\Delta \mu=2 \mathrm{MeV}$ soon after recombination, electrons of typical kinetic energy $\sim \mathrm{MeV}$ would be produced, each of which can reionize $\sim 10^{5} \mathrm{H}$ atoms. Given that $\rho_{\mathrm{DM}} / \rho_{H} \approx 5$ and that each DM state undergoes $\mathcal{O}(1)$ decays per Hubble time as explained above, the hydrogen would be quickly completely reionized, in gross conflict with $\mathrm{CMB}$ observations. Even decays close to kinematic threshold are ruled out. To avoid this constraint, we require that decays into $e^{+} e^{-}$pairs become kinematically impossible at or before the CMB decoupling time $t_{\mathrm{CMB}}$. We conservatively estimate $\Delta \mu_{\mathrm{CMB}}$ using $\Gamma=$ $H\left(t_{\mathrm{CMB}}\right)$ (where $\Gamma$ is the total $\mathrm{DM}$ decay rate including neutrino final states), and require $\Delta \mu_{\mathrm{CMB}}<2 m_{e} \approx 1 \mathrm{MeV}$. (In the Supplemental Material [49], we show that this simple estimate is supported by a more detailed analysis using numerically calculated DM mass distribution.) Another constraint arises from the photodissociation of nuclei due to electromagnetic energy injected by DM decays, which can change their primordial abundances and spoil the successful predictions of big-bang nucleosynthesis (BBN) [10-15]. The photodissociation becomes relevant when the temperature $T<0.5 \mathrm{keV}$, which gives a weaker bound than the $\mathrm{CMB}$. The $\mathrm{CMB}$ and $\mathrm{BBN}$ constraints give lower bounds on the mixing angle $\sin \alpha$, shown by the blue and magenta curves in Fig. 1 .
After recombination, DM states continue to decay via the neutrino channel. Neutrinos from such decays could in principle be detected, but the predicted flux is well below the current constraints [42-45].

Direct detection.-Direct detection signature of WIC is due to a scattering process $\mathrm{DM}(\mu)+N \rightarrow \operatorname{DM}\left(\mu^{\prime}\right)+N$, where $N$ is a target nucleus. Since $\mu$ and $\mu^{\prime}$ are continuous variables, the kinematics of this process in the WIC model is quite distinct from either elastic or inelastic particle DM. This unique kinematics leads to a strong suppression of the direct detection rate, which is one of the most striking features of such models, and allows for a viable $Z$-portal WIC model consistent with current bounds.

The direct detection rate for the continuum DM is

$$
\frac{d R}{d E_{R}}=N_{T} \int \frac{d \mu^{2}}{2 \pi} \nu_{0}\left(\mu^{2}\right) \int d^{3} v f(v) \frac{d \sigma}{d E_{R}} v
$$

where $E_{R}$ is the recoil energy of the nucleus, $N_{T}$ the number of nuclei per unit mass, $\nu_{0}$ is the current WIC mass distribution function, and $f(v)$ is the local DM velocity distribution. We use the approximation

$$
\nu_{0}=\frac{\rho_{\odot}}{\mu_{0}} \delta\left(\mu^{2}-\mu_{1}^{2}\right)
$$

where $\rho_{\odot}=0.3 \mathrm{GeV} / \mathrm{cm}^{3}$ is the local DM density, and $\mu_{1}$ is found by equating the WIC decay rate with today's Hubble scale. (For typical parameters, $\mu_{1}-\mu_{0} \sim 100-300 \mathrm{keV}$.) We assume the Maxwell-Boltzmann distribution for the WIC DM with a cutoff at the escape velocity $v_{\text {esc }}=600 \mathrm{~km} / \mathrm{s}$. Since the DM state masses today are clustered near the gap scale with a small relative spread, the velocity distribution is approximately mass independent. The differential cross section is

$\frac{d \sigma}{d E_{R}}=\int_{\mu_{0}^{2}}^{\mu_{\max }^{2}} \frac{d \mu^{\prime 2}}{2 \pi} \rho\left(\mu^{\prime 2}\right) \frac{m_{N} \sigma_{p}}{2 \mu_{\Phi n}^{2} v^{2}}\left[\frac{f_{p} Z+f_{n}(A-Z)}{f_{p}}\right]^{2} F_{N}^{2}(q)$.

The DM-proton (or neutron) cross section is

$$
\sigma_{p}=\frac{\left(g^{2}+g^{\prime 2}\right)^{2} \sin ^{4} \alpha \mu_{\Phi n}^{2} f_{p}^{2}}{16 \pi m_{Z}^{4}} .
$$

Here, $\mu_{\Phi n}$ is the reduced mass of the DM state and proton or neutron, and $F_{N}(q)$ is the nucleus form factor [46]. The coupling strength to nucleon $f_{p}\left(f_{n}\right)$ is given by the vector current matching from the quark to nucleon, $f_{n}=$ $b_{u}+2 b_{d}=-\frac{1}{4}$ and $f_{p}=2 b_{u}+b_{d}=\frac{1}{4}-\sin ^{2} \theta_{w}$. The incoming DM state with mass $\mu_{1}$ can be down-scattered or up-scattered. Using energy-momentum conservation, the maximum accessible mass for the outgoing state is 


$$
\mu_{\max }=\mu_{1}+q v-\frac{1}{2} \frac{q^{2}}{\mu_{\Phi N}},
$$

where $q=\sqrt{2 m_{N} E_{R}}$ is the exchanged momentum. Since $\mu_{1}-\mu_{0} \ll \mu_{0}$ and $v \ll 1$, only a narrow range of DM states are kinematically accessible, suppressing the cross section by

$$
\int_{\mu_{0}^{2}}^{\mu_{\max }^{2}} \frac{d \mu^{\prime 2}}{2 \pi} \rho\left(\mu^{\prime 2}\right) \sim\left(\frac{\Delta \mu}{\mu_{0}}\right)^{3 / 2} \sim \mathcal{O}\left(10^{-6}-10^{-7}\right) .
$$

The direct detection bound was obtained by comparing the predicted number of events in the Z-portal WIC model with the corresponding particle DM prediction, and recasting the bounds from the XENON1T experiment [5]. Thanks to the strong continuum kinematic suppression, XENON1T limits do not impose a constraint for $\rho_{0}=1$. (Direct detection constraints become relevant for larger $\rho_{0}$; see Supplemental Material [49].) For comparison, the limit on particle scalar Z-portal DM is shown by the dotted cyan curve in Fig. 1.

Other constraints.-Indirect detection does not currently constrain the WIC model. The kinematics and the cross section of DM annihilation in today's halos is virtually identical to usual particle DM of mass $\mu_{0}$. For Z-portal DM with $\mu_{0}<m_{W}$, the dominant channel is $\Phi \Phi^{*} \rightarrow Z^{*} \rightarrow f \bar{f}$, and the rate is $p$-wave suppressed. For $\mu_{0}>m_{W}$, DM can annihilate via $s$ wave into $W W$. The rate is below the current bounds from FermiLAT [47].

At colliders, continuous DM states can be pair produced through virtual $Z$ exchange. Each produced DM state decays to a lighter DM state plus on- or off-shell $Z$. The lighter DM state in turn undergoes further decays, until the DM cascades down to a state so close to the gap that its decays are invisible in the detector due to its long lifetime and/or softness of its SM decay products. Overall, the event looks similar to the $X+$ MET signature of neutralino $\tilde{\chi}_{2}^{0}$ production in SUSY models, but with higher multiplicity and somewhat softer spectrum of visible particles. Continuum DM production cross sections are suppressed by a factor of $\sin ^{4} \alpha \sim 10^{-2}$ compared to SM electroweak processes, in addition to continuum kinematic suppression near the mass threshold similar to the suppression of direct detection rates. As a result, the $Z$-portal DM model is consistent with current LHC bounds. For $\mu_{0}<m_{Z} / 2$, where $Z$ decays to on-shell DM pairs are allowed, strong bounds from LEP-1 and SLD become relevant. However, the bounds on WIC are again much weaker compared to the particle Z-portal DM, due to the continuum kinematic suppression of the decay rate. We conservatively estimate the bound by comparing the rate to the experimental limit on the invisible $Z$ decays $\Gamma_{\text {inv }}$ [48]. The resulting bound is quite weak, and does not place a relevant constraint for $\rho_{0}=1$. For $\mu_{0}<m_{h} / 2$ a Higgs decay to DM pairs would produce exotic semi-invisible final states. Demanding that no more than $10 \%$ of Higgs bosons decay in this channel yields a bound indicated by the gray line in Fig. 1. We will explore collider phenomenology of the Z-portal WIC model in detail in future work.

Conclusions. - In this Letter, we initiated the study of phenomenology of the Z-portal WIC model introduced in the companion paper [16]. In this model the role of DM is played by a gapped continuum, rather than an ordinary particle, which acquires couplings to the $\mathrm{SM} Z / W$ bosons from mixing with the Higgs. When the temperature is below the gap scale $\mu_{0}$, the WIC mass distribution is strongly peaked around $\mu_{0}$, and the thermal freeze-out of WICs is essentially identical to that of ordinary particle WIMPs. However, other properties of the WIC are strikingly different. Because of the unique features of continuum kinematics, the direct detection cross section is strongly suppressed, reopening the $Z$ portal for WICs. WIC decays $\Phi\left(\mu_{1}\right) \rightarrow \Phi\left(\mu_{2}\right)+$ SM occur throughout the history of the Universe, with each DM state decaying on average once per Hubble time. Such decays may yield new sources of $e^{+} e^{-}$pairs during and after recombination, resulting in a lower bound on the WIC coupling to the SM. Collider signals of WICs include a cascade of SM particles and the invisible long-lived WIC states. Our analysis demonstrated that the Z-portal WIC provides a fully viable DM candidate consistent with all experimental constraints, as shown in the summary plot in Fig. 1. We look forward to further studies of the rich and novel phenomenology of these models.

We thank Francesca Calore, Barry McCoy, Eun-Gook Moon, Carlos Wagner, Liantao Wang, and Kathryn Zurek for useful discussions. C. C., S. H., G. K., and M. P. were supported in part by the NSF Grant No. PHY-2014071. C. C. was also supported in part by the U.S.-Israeli BSF Grant No. 2016153. S. H. was also supported by the DOE Grants No. DE-SC-0013642 and No. DE-AC0206CH11357, and by Hans Bethe Postdoctoral fellowship at Cornell. G. K. is supported by the Science and Technology Facilities Council with Grant No. ST/ T000864/1. S. L. was supported by the Samsung Science and Technology Foundation and by the National Research Foundation of Korea (NRF) grant funded by the Korea government (MEST) (No. NRF-2021R1A2C1005615). W.X. was supported in part by the DOE Grant No. DE-SC0010296.

[1] B. W. Lee and S. Weinberg, Cosmological Lower Bound on Heavy Neutrino Masses, Phys. Rev. Lett. 39, 165 (1977).

[2] G. Jungman, M. Kamionkowski, and K. Griest, Supersymmetric dark matter, Phys. Rep. 267, 195 (1996).

[3] G. Bertone, D. Hooper, and J. Silk, Particle dark matter: Evidence, candidates and constraints, Phys. Rep. 405, 279 (2005). 
[4] M. Escudero, A. Berlin, D. Hooper, and M.-X. Lin, Toward (finally!) ruling out $Z$ and Higgs mediated dark matter models, J. Cosmol. Astropart. Phys. 12 (2016) 029.

[5] E. Aprile et al. (XENON Collaboration), Dark Matter Search Results from a One Ton-Year Exposure of XENON1T, Phys. Rev. Lett. 121, 111302 (2018).

[6] D. S. Akerib et al. (LUX Collaboration), Results from a Search for Dark Matter in the Complete LUX Exposure, Phys. Rev. Lett. 118, 021303 (2017).

[7] X. Cui et al. (PandaX-II Collaboration), Dark Matter Results From 54-Ton-Day Exposure of PandaX-II Experiment, Phys. Rev. Lett. 119, 181302 (2017).

[8] P. A. R. Ade et al. (Planck Collaboration), Planck 2015 results. XIII. Cosmological parameters, Astron. Astrophys. 594, A13 (2016).

[9] T. R. Slatyer, Indirect dark matter signatures in the cosmic dark ages. I. Generalizing the bound on $s$-wave dark matter annihilation from Planck results, Phys. Rev. D 93, 023527 (2016).

[10] M. H. Reno and D. Seckel, Primordial nucleosynthesis: The effects of injecting hadrons, Phys. Rev. D 37, 3441 (1988).

[11] M. Kawasaki and T. Moroi, Electromagnetic cascade in the early Universe and its application to the big bang nucleosynthesis, Astrophys. J. 452, 506 (1995).

[12] R. H. Cyburt, J. R. Ellis, B. D. Fields, and K. A. Olive, Updated nucleosynthesis constraints on unstable relic particles, Phys. Rev. D 67, 103521 (2003).

[13] M. Kawasaki, K. Kohri, and T. Moroi, Big-bang nucleosynthesis and hadronic decay of long-lived massive particles, Phys. Rev. D 71, 083502 (2005).

[14] J. Hisano, M. Kawasaki, K. Kohri, T. Moroi, and K. Nakayama, Cosmic rays from dark matter annihilation and big-bang nucleosynthesis, Phys. Rev. D 79, 083522 (2009).

[15] B. Henning and H. Murayama, Constraints on light dark matter from big bang nucleosynthesis, arXiv:1205.6479.

[16] C. Csáki, S. Hong, G. Kurup, S. J. Lee, M. Perelstein, and W. Xue, companion paper, Continuum dark matter, Phys. Rev. D 105, 035025 (2022).

[17] Our model shares some features with dynamical DM models proposed in [18-27], but the two frameworks also have important differences in both model-building and phenomenological predictions. For a detailed comparison, see Ref. [16]. Also, in [28-30], a continuum is used as the mediator to the DM, while here the continuum is the DM itself.

[18] K. R. Dienes and B. Thomas, Dynamical dark matter: I. Theoretical overview, Phys. Rev. D 85, 083523 (2012).

[19] K. R. Dienes and B. Thomas, Dynamical dark matter: II. An explicit model, Phys. Rev. D 85, 083524 (2012).

[20] K. R. Dienes, S. Su, and B. Thomas, Distinguishing dynamical dark matter at the LHC, Phys. Rev. D 86, 054008 (2012).

[21] K. R. Dienes, J. Kumar, and B. Thomas, Direct detection of dynamical dark matter, Phys. Rev. D 86, 055016 (2012).

[22] K. R. Dienes, J. Kumar, B. Thomas, and D. Yaylali, Overcoming velocity suppression in dark-matter direct-detection experiments, Phys. Rev. D 90, 015012 (2014).

[23] K. R. Dienes, J. Kumar, B. Thomas, and D. Yaylali, DarkMatter Decay as a Complementary Probe of Multi- component Dark Sectors, Phys. Rev. Lett. 114, 051301 (2015).

[24] K. R. Dienes, S. Su, and B. Thomas, Strategies for probing nonminimal dark sectors at colliders: The interplay between cuts and kinematic distributions, Phys. Rev. D 91, 054002 (2015).

[25] K. K. Boddy, K. R. Dienes, D. Kim, J. Kumar, J.-C. Park, and B. Thomas, Lines and boxes: Unmasking dynamical dark matter through correlations in the $\mathrm{MeV} \gamma$-ray spectrum, Phys. Rev. D 94, 095027 (2016).

[26] D. Curtin, K. R. Dienes, and B. Thomas, Dynamical dark matter, MATHUSLA, and the lifetime frontier, Phys. Rev. D 98, 115005 (2018).

[27] K. R. Dienes, D. Kim, H. Song, S. Su, B. Thomas, and D. Yaylali, Nonminimal dark sectors: Mediator-induced decay chains and multijet collider signatures, Phys. Rev. D 101, 075024 (2020).

[28] B. von Harling and K. L. McDonald, Secluded dark matter coupled to a hidden CFT, J. High Energy Phys. 08 (2012) 048.

[29] A. Katz, M. Reece, and A. Sajjad, Continuum-mediated dark matter-baryon scattering, Phys. Dark Universe 12, 24 (2016).

[30] I. Chaffey, S. Fichet, and P. Tanedo, Continuum-mediated self-interacting dark matter, J. High Energy Phys. 06 (2021) 008.

[31] H. Georgi, Unparticle Physics, Phys. Rev. Lett. 98, 221601 (2007).

[32] D. Stancato and J. Terning, The unhiggs, J. High Energy Phys. 11 (2009) 101.

[33] A. Falkowski and M. Perez-Victoria, Holographic unhiggs, Phys. Rev. D 79, 035005 (2009).

[34] B. Bellazzini, C. Csáki, J. Hubisz, S. J. Lee, J. Serra, and J. Terning, Quantum Critical Higgs, Phys. Rev. X 6, 041050 (2016).

[35] C. Csáki, G. Lee, S. J. Lee, S. Lombardo, and O. Telem, Continuum naturalness, J. High Energy Phys. 03 (2019) 142.

[36] S. S. Gubser, Curvature singularities: The good, the bad, and the naked, Adv. Theor. Math. Phys. 4, 679 (2000).

[37] S. Sachdev, Quantum phase transitions, Handbook of Magnetism and Advanced Magnetic Materials (Wiley, Hoboken, 2007).

[38] E. H. Fradkin, Field Theories of Condensed Matter Physics (Cambridge University Press, Cambridge, England, 2013), Vol. 82.

[39] B. M. McCoy and T. T. Wu, Two-dimensional Ising field theory in a magnetic field: Breakup of the cut in the two point function, Phys. Rev. D 18, 1259 (1978).

[40] L. Randall and R. Sundrum, A Large Mass Hierarchy from a Small Extra Dimension, Phys. Rev. Lett. 83, 3370 (1999).

[41] J. A. Cabrer, G. von Gersdorff, and M. Quiros, Soft-wall stabilization, New J. Phys. 12, 075012 (2010).

[42] E. Aprile et al. (XENON Collaboration), Excess electronic recoil events in XENON1T, Phys. Rev. D 102, 072004 (2020).

[43] K. Bays et al. (Super-Kamiokande Collaboration), Supernova relic neutrino search at super-kamiokande, Phys. Rev. D 85, 052007 (2012). 
[44] M. Agostini et al. (BOREXINO Collaboration), Sensitivity to neutrinos from the solar CNO cycle in Borexino, Eur. Phys. J. C 80, 1091 (2020).

[45] M. Agostini et al. (BOREXINO Collaboration), Experimental evidence of neutrinos produced in the CNO fusion cycle in the Sun, Nature (London) 587, 577 (2020).

[46] J. D. Lewin and P. F. Smith, Review of mathematics, numerical factors, and corrections for dark matter experiments based on elastic nuclear recoil, Astropart. Phys. 6, 87 (1996).

[47] A. Albert et al. (Fermi-LAT, DES Collaboration), Searching for dark matter annihilation in recently discovered Milky Way satellites with Fermi-LAT, Astrophys. J. 834, 110 (2017).
[48] P. Zyla et al. (Particle Data Group), Review of particle physics, Prog. Theor. Exp. Phys. 2020, 083 C01 (2020).

[49] See Supplemental Material at http://link.aps.org/ supplemental/10.1103/PhysRevLett.128.081807 which includes [50,51], for out-of-equilibrium decays, electromagnetic energy injection after recombination, and effect of varying spectral density amplitude near the mass gap.

[50] T. R. Slatyer and C.-L. Wu, General constraints on dark matter decay from the cosmic microwave background, Phys. Rev. D 95, 023010 (2017).

[51] V. Poulin, J. Lesgourgues, and P. D. Serpico, Cosmological constraints on exotic injection of electromagnetic energy, J. Cosmol. Astropart. Phys. 03 (2017) 043. 